\title{
BMJ Open Systemic immunomodulatory treatments for atopic dermatitis: protocol for a systematic review with network meta-analysis
}

\author{
Aaron Mark Drucker, ${ }^{1,2}$ Alexandra Ellis, ${ }^{3}$ Zarif Jabbar-Lopez, ${ }^{4}$ Zenas Z N Yiu, ${ }^{5}$ \\ Bernd W M Arents, ${ }^{6}$ Tim Burton, ${ }^{7}$ Phyllis I Spuls, ${ }^{8}$ Denise Küster, ${ }^{9}$ Jochen Schmitt, ${ }^{9}$ \\ Carsten Flohr ${ }^{4}$
}

To cite: Drucker AM, Ellis A, Jabbar-Lopez Z, et al. Systemic immunomodulatory treatments for atopic dermatitis: protocol for a systematic review with network meta-analysis. BMJ Open 2018;8:e023061. doi:10.1136/ bmjopen-2018-023061

- Prepublication history for this paper is available online. To view these files, please visit the journal online (http://dx.doi org/10.1136/bmjopen-2018023061).

Received 20 March 2018 Revised 6 July 2018 Accepted 20 July 2018

Check for updates

(c) Author(s) (or their employer(s)) 2018. Re-use permitted under CC BY-NC. No commercial re-use. See rights and permissions. Published by BMJ.

For numbered affiliations see end of article.

Correspondence to Dr Aaron Mark Drucker; aaron.drucker@wchospital.ca

\section{ABSTRACT}

Introduction There are numerous new systemic treatments for atopic dermatitis in various stages of development and most are being compared with placebo rather than active comparators. In order to understand the relative efficacy and safety of existing and new treatments for atopic dermatitis, robust mixed comparisons (ie, direct and indirect) would be beneficial. To address this gap, this protocol describes methods for a systematic review and network meta-analysis of systemic treatments for atopic dermatitis.

Methods and analysis We will update the search of a previous systematic review, including searches of the Cochrane Central Register of Controlled Trials, MEDLINE, Embase, Latin American and Caribbean Health Science Information database and the Global Resource of EczemA Trials database in addition to clinical trial protocol registries. Title, abstract and full paper screening as well as data extraction will be conducted in duplicate by independent researchers. Primary outcomes include efficacy with regards to clinician-reported signs and patient-reported symptoms and safety with regards to withdrawal from treatment due to adverse events and the occurrence of serious adverse events. Secondary outcomes will include change in quality of life and itch severity. Where possible and appropriate, network metaanalysis will be performed for each outcome using a random-effects model within a Bayesian framework. If appropriate, the review will be transitioned to a living review with continuous updating of the analysis.

Ethics and dissemination Dissemination in a peerreviewed scientific journal is planned.

PROSPERO registration number CRD42018088112;

Pre-results.

\section{INTRODUCTION}

Atopic dermatitis (AD) is a chronically relapsing inflammatory skin condition. For most patients, $\mathrm{AD}$ is mild and can be managed effectively with over-the-counter emollients and prescription topical therapies including corticosteroids. It is estimated that $7 \%$ of children and $2 \%-8 \%$ of adults with $\mathrm{AD}$ have
Strengths and limitations of this study

- We will conduct a thorough literature search to identify all relevant trials on the efficacy and safety of systemic treatments for atopic dermatitis, building on a Cochrane review that does not incorporate quantitative synthesis.

- The efficacy outcomes of interest represent three important domains, namely change in clinician-reported signs of disease, patient-reported symptoms and patient-reported quality of life.

- Network meta-analysis, if appropriate, will allow comparison of treatments that have not been compared head to head.

- Diverse outcome measurement instruments used to assess the three outcome domains and other differences in trial design may limit our ability to pool results from different studies.

- The study team includes patients, clinicians and methodologists.

severe disease. ${ }^{12}$ For these patients, topical therapies may be unsuccessful or inadequate, and treatment with phototherapy or systemic therapy may be warranted. ${ }^{3}$

For years, systemic therapeutic options were limited to traditional immunosuppressive medications such as ciclosporin, methotrexate, azathioprine, mycophenolate and corticosteroids. $^{4}$ More recently, targeted agents have been developed including dupilumab, the first biological approved for the treatment of moderate-to-severe $\mathrm{AD} .^{5}$ Many other biological and small-molecule treatments are currently being tested in clinical trials. ${ }^{6}$

Determining the relative efficacy and safety of the older and newer systemic therapies for $\mathrm{AD}$ is challenging. Most randomised controlled trials (RCTs) do not use standardised outcome measures and head-tohead comparison are rare. ${ }^{45-13}$ Therefore, 
Table 1 Specific objectives (participants, interventions, comparators, outcomes and design).

\begin{tabular}{|c|c|}
\hline Participants & $\begin{array}{l}\text { Patients (children and adults) with } \\
\text { moderate-to-severe atopic dermatitis }\end{array}$ \\
\hline Interventions & $\begin{array}{l}\text { Systemic immunomodulatory agents, } \\
\text { including: } \\
\text { Ciclosporin. } \\
\text { Methotrexate. } \\
\text { Azathioprine. } \\
\text { Mycophenolate. } \\
\text { Corticosteroids. } \\
\text { Dupilumab. } \\
\text { Nemolizumab. } \\
\text { Lebrikizumab. } \\
\text { Ustekinumab. } \\
\text { Fezakinumab. } \\
\text { Baricitinib. } \\
\text { Apremilast. } \\
\text { Interferon. } \\
\text { Intravenous immunoglobulin. } \\
\text { Others, including new agents whose } \\
\text { first trials are published between } \\
\text { publication of this protocol and our final } \\
\text { literature search. }\end{array}$ \\
\hline Comparators & Any, including placebo. \\
\hline Outcomes & $\begin{array}{l}\text { Primary outcomes - efficacy } \\
\text { 1. Change in investigator-reported clinical } \\
\text { signs (eg, EASI and o-SCORAD). } \\
\text { 2. Change in patient-reported symptoms } \\
\text { (eg, POEM). } \\
\text { Primary outcomes - safety } \\
\text { 3. Withdrawal from systemic treatment } \\
\text { due to adverse events. } \\
\text { 4. Occurrence of serious adverse events. } \\
\text { Secondary outcomes } \\
\text { 5. Change in health-related quality of life } \\
\text { (eg, DLQI). } \\
\text { 6. Change in itch severity. }\end{array}$ \\
\hline Design & Randomised controlled trials. \\
\hline
\end{tabular}

DLQI, Dermatology Life Quality Index; EASI, Eczema Area Severity Index; o-SCORAD, objective SCORAD; POEM, Patient Oriented Eczema Measure.

in order for clinicians and patients to understand how established and upcoming therapies compare with regards to efficacy and safety, indirect comparisons must be made. The aim of our study is to conduct a systematic review and network meta-analysis (NMA) to determine the relative efficacy and safety of systemic treatments for $\mathrm{AD}$ (table 1). To date, no NMA has been conducted comparing systemic treatments for $\mathrm{AD}$.

\section{METHODS AND ANALYSIS}

This protocol has been written according to the Preferred Reporting Items for Systematic review and Meta-Analysis Protocols guidance ${ }^{14}$ and has been registered on PROSPERO (http://www.crd.york.ac.uk/PROSPERO/display_ record.php?ID=CRD42018088112). The research team consists of patients with $\mathrm{AD}$, clinicians and methodologists, all of whom have contributed to the design of this study. The specific research objectives are summarised in table 1 .

\section{Eligibility criteria}

All RCTs of immunomodulatory systemic therapies for moderate-to-severe $\mathrm{AD}$ will be included in this review, without age and sex restriction. Due to the absence of an established definition of moderate-to-severe AD, RCTs will be eligible when including subjects defined as: 'patients with moderate-to-severe AD', 'patients with non-adequately controlled $\mathrm{AD}$ despite the use of topical anti-inflammatory therapy' or patients with moderate-to-severe $\mathrm{AD}$ according to published severity criteria. ${ }^{15} 16$ We will summarise the inclusion criteria used for each study. All other study types and disease states will be excluded, including studies on other forms of eczema/dermatitis such as chronic hand dermatitis.

RCTs that compare systemic immunomodulatory therapies for $\mathrm{AD}$ with any comparator, including placebo, are eligible. Systemic immunomodulatory therapies include ciclosporin, methotrexate, azathioprine, mycophenolate, corticosteroids, interferon-gamma, intravenous immunoglobulin, dupilumab and other novel systemic agents. We will include studies with systemic immunomodulatory therapies as monotherapy or in combination with topical therapies. Medications used at different dosages will be treated as separately in the primary network meta-analysis. Studies investigating other systemic therapies, such as Chinese herbal remedies, antihistamines, leukotriene antagonists, oral calcineurin inhibitors, vaccinations, phototherapy or antiviral/antibiotic agents will not be considered.

In order to be included, RCTs must report sufficient data on at least one of the primary or secondary outcomes listed in table 1. Sufficient data include a point estimate and a measure of variance (eg, SE and 95\% CI) for continuous outcomes and sample size with number of patients experiencing an event for binary outcomes. We will examine these endpoints for short-term ( $\leq 16$ weeks) and long-term ( $>16$ weeks) treatment.

\section{Information sources and search strategy}

Our searches will update those of a Cochrane review without quantitative synthesis authored by members of our research team. ${ }^{17}$ Electronic searches will be performed in the following databases: Cochrane Central Register of Controlled Trials (CENTRAL), MEDLINE via Ovid (from 1946), Embase via Ovid (from 1974), Latin American and Caribbean Health Science Information database (from 1982) and the Global Resource of EczemA Trials database. Our search strategies for these databases will be modelled on the MEDLINE strategy originally developed for the previous Cochrane systematic review. ${ }^{17}$ Searches will also be performed in the following trials registers: the ISRCTN registry (www.isrctn.com), ClinicalTrials. gov (www.ClinicalTrials.gov), the Australian New Zealand 
Clinical Trials Registry (www.anzctr.org.au), the WHO International Clinical Trials Registry Platform and the EU Clinical Trials Register (www.clinicaltrialsregister.eu).

We will hand search reference lists of relevant publications that are retrieved as full papers as well as relevant systematic reviews and literature reviews to identify other eligible studies. Experts in the field will be contacted for additional published and unpublished studies.

We will include data from published peer-reviewed journals, conference abstracts, trial registries and product monographs. Only studies published in English will be included, as language restriction has been shown not to bias the results of quantitative syntheses. ${ }^{18}$ We anticipate that the language of publication will not be differential with regards to treatment outcomes, so it is unlikely to bias our results. We will not place any restriction on publication year.

\section{Study records}

This systematic review will build on the results of the Cochrane systematic review. ${ }^{17}$ The results of updated searches will be uploaded into Abstrackr (http:// abstrackr.cebm.brown.edu/) for title and abstract screening. ${ }^{19}$ Two independent researchers will screen titles and abstracts of papers, eliminating those deemed irrelevant. A third researcher will resolve discrepancies. Two independent researchers will read each potentially relevant paper in full, selecting papers meeting specific inclusion criteria as above.

Two researchers will independently extract data from each included trial, using the data extraction form from the previous review. ${ }^{17}$ The full list of data to be extracted has been previously published. In brief, we will extract general characteristics of the publication, study date and setting, participant characteristics (age, sex and AD severity), inclusion and exclusion criteria, descriptions of interventions and outcomes data. To our knowledge, trial reports of systemic therapy for $\mathrm{AD}$ have not included individual patient-level data. As such, data will be extracted at the trial arm level, rather than the individual patient level. If, in the future, individual patient data becomes more readily available for relevant trials, incorporating such individual patient data could improve the precision of the NMA. ${ }^{20}$

\section{Outcomes}

The Harmonizing Outcome Measures for Eczema (HOME) initiative has identified clinician-reported signs, patient-reported symptoms, quality of life and long-term control as core domains for assessment in RCTs for AD. ${ }^{21}$ HOME aims to identify individual outcome measures to be used in all RCTs and has selected the Eczema Area Severity Index (EASI) for signs ${ }^{22}$ and Patient Oriented Eczema Measure (POEM) for symptoms. ${ }^{23}$ No core instruments have been selected for quality of life and long-term control, and long-term control is generally not measured as a separate domain in most RCTs. Unfortunately, most RCTs for $\mathrm{AD}$ predate HOME, and as such outcome measures are not standardised across RCTs. ${ }^{4}$ Therefore, we will extract data on all measures of signs, symptoms and quality of life.

The two most commonly used measures for clinical signs in AD RCTs are EASI and objective SCORAD (Scoring Atopic Dermatitis; o-SCORAD), and they each have reasonable measurement properties. ${ }^{24}{ }^{25}$ As EASI was selected by HOME as the core outcome for clinical signs, it will be prioritised as the preferred outcome measure in our analysis. Similarly, the POEM scale will be used as the primary measure of $\mathrm{AD}$ symptoms. The most prominent symptoms of $\mathrm{AD}$ is itch, and separate measurement of change in itch severity will be extracted as a secondary outcome where available. The Dermatology Life Quality Index is the most commonly used instrument for quality of life in $\mathrm{RCTs}^{26}$; therefore, despite inadequate evidence for strong measurement properties, it will be prioritised in our analysis. ${ }^{26}$

For each efficacy outcome, we will extract means and SEs for each study arm. Where SD or CIs are reported, these will be transformed to SEs. Authors of studies that do not report these outcomes as continuous variables or that do not report SD/SE will be contacted for this information. Where SD/SE data are not available, the mean value of known SDs will be imputed from the group of included studies. ${ }^{27}$

The two included safety outcomes are withdrawal from treatment due to adverse events (tolerability) and the occurrence of serious adverse events. For these outcomes, we will rely on reporting of these terms in the trial publications. Where adverse event rates in those specific categories are not given in the report, we will contact the authors for that data. For each safety outcome, we will extract the sample size of each treatment and the number of patients experiencing the event.

\section{Data synthesis}

Where possible, we intend to synthesise study data using NMAs. NMA is an extension of pairwise meta-analysis that simultaneously combines both the direct evidence (ie, interventions assessed head to head) and indirect evidence (ie, interventions assessed through a common comparator) ${ }^{28}{ }^{29}$ Doing so improves precision of treatment effect estimates and also provides estimates for all pairwise comparisons including those missing from the direct evidence. ${ }^{29} 30$

For each outcome, NMA will be conducted when there are sufficiently similar studies forming a network (ie, the studies within the set share at least one common treatment). Within each outcome domain (eg, clinical signs), we plan to analyse each scale (eg, EASI and o-SCORAD) separately. In a separate analysis, we also plan to combine all scales within an outcome domain using standardised mean differences.

NMA will be performed using a random-effects model within a Bayesian framework using the gemtc R package. ${ }^{31}$ For continuous outcomes (eg, change in clinical signs), the NMA model corresponds to a generalised linear 
model with identity link. ${ }^{32}$ For binary outcomes (eg, adverse events), the NMA model corresponds to a generalised linear model with logit link. ${ }^{32}$ We will include random effects on the treatment parameters, which allows each study to have a different but related treatment effect. The between-study variance (heterogeneity) will be assumed to be constant for every treatment comparison. We will use non-informative prior distributions for all model parameters. Convergence of four chains will be assessed by the Gelman-Rubin statistic and visual inspection of trace plots.

Two key assumptions of NMA are transitivity and consistency. Transitivity relates to the validity of estimating an unobserved direct comparison through the available indirect evidence. Although transitivity cannot be tested statistically, its plausibility can be conceptually evaluated. The restriction of our analysis to include only studies of moderate to severe $\mathrm{AD}$ makes our transitivity assumption plausible. However, this will be evaluated further by examining the distribution of other baseline factors that may influence treatment response, such as concomitant topical therapy, duration of $\mathrm{AD}$, baseline $\mathrm{AD}$ severity and age. Consistency extends the assumption of transitivity to 'loops' of evidence and relates to the agreement of the direct and indirect estimates. For each analysis, we will empirically assess the consistency of the network by comparing the direct and indirect evidence using a node-splitting approach. ${ }^{33}$ This approach estimates the direct and indirect treatment effect estimates separately. Discrepancies between these estimates indicate inconsistency. If there is evidence of inconsistency, only the results of the direct comparisons will be presented.

In addition to summary results presented as an OR or mean with a $95 \%$ credible interval, the cumulative rankings of treatments will also be presented. Cumulative ranking probability plots represent the ranking probabilities of the various treatments with a visual estimation of their uncertainty. Rankings will be quantified by the Surface Under the Cumulative Ranking (SUCRA) that express the percentage $(0 \%-100 \%)$ of efficacy/safety each treatment has compared with an ideal treatment ranked always first without uncertainty. ${ }^{34}$ The larger the SUCRA value, the better the rank.

\section{Subgroup and sensitivity analyses}

The robustness of the primary efficacy and safety estimates from the NMA will be evaluated by analysing only outcomes with low risk of bias (as defined below). Subgroup analyses will also be conducted for children and adults. Outcome data on short-term $(\leq 16$ weeks) as well as long-term/maintenance ( $>16$ weeks) treatment will be analysed separately. We chose this cut-off as most trials of systemic treatments for $\mathrm{AD}$ are 12-16 weeks in length. While the HOME group has included long-term control as a core outcome domain for clinical trials, we will most likely not be able to assess true long-term control in our analysis, as this is unfortunately rarely assessed in clinical trials. $^{21}$

\section{Assessment of bias and strength of evidence}

Two independent researchers will assess the risk of bias in individual studies using the Cochrane Risk of Bias 2.0 tool. ${ }^{35}$ To empirically assess for publication bias, we will compare the results of our trial registry searches with the results from published studies. We will further assess for reporting bias by comparing the outcomes prespecified in the trial registries with the reported outcomes. We will assess the overall quality of evidence for each outcome using the Grading of Recommendations Assessment, Development and Evaluation criteria. ${ }^{36}$

\section{Updating}

A recent study concluded that living network meta-analyses with continuous updating produce strong, timely evidence of comparative effectiveness. ${ }^{37}$ The research questions in this systematic review are in line with proposed criteria for continuing a living systematic review, namely: (1) the systematic review is a priority for decision making; (2) new information will change decision making; and (3) there is likely to be, on an ongoing basis, new research relevant to decision making. ${ }^{38} 39$ As such, if these criteria are still met at the conclusion of our baseline review and analysis, we will convert the review to a living systematic review with network meta-analysis. Given the number of new systemic medications in development for atopic dermatitis, this is likely to be the case. ${ }^{6}$

Updated searches will be conducted monthly, with relevant studies added to the review. The analysis will be updated every 4 months at a minimum but will be updated more frequently if new studies meet any of the following three conditions:

1. Newly identified studies include outcomes data on a new systemic medication not currently included in the network meta-analysis.

2. Newly identified studies include comparisons between medications that have never before been directly compared.

3. Results of newly identified studies are inconsistent with the results of the most recent network meta-analysis (eg, if in the most recent network meta-analysis methotrexate is superior at improving symptoms compared with ciclosporin, but in a newly identified clinical trial ciclosporin is found to be superior).

\section{Patient and public involvement}

Our research team includes atopic dermatitis patients, one of whom represents the Dutch Association for People with Atopic Dermatitis (VMCE), a patient advocacy group. They have contributed to the development of this protocol including the selection of outcomes of importance to patients. They will continue to contribute to the study going forward, ensuring that our results are presented in a way that is meaningful to patient decision making. 


\section{ETHICS AND DISSEMINATION}

There is no primary data collection involved in this study, so research ethics approval is not required.

We plan to disseminate our results through publication in a peer-reviewed scientific journal. We will report our results following the framework laid out in the Preferred Reporting Items for Systematic Reviews and Meta-Analyses extension for NMA. ${ }^{40}$

\section{SUMMARY}

In this systematic review and network meta-analysis, we will provide the first comprehensive quantitative synthesis of systemic treatments for AD. As new systemic treatments are developed and tested clinically, with some eventually obtaining clinical approval, it will be essential to compare new and established treatments in a rigorous manner. Ideally, new treatments for $\mathrm{AD}$ will be assessed against existing treatments in head-to-head RCTs. However, this is unlikely to be the case. Using psoriasis as an example, most new systemic agents are only compared with placebo and, sometimes, a single active comparator. Recent NMAs for psoriasis have provided a solution, giving patients, clinicians and other stakeholders a means of comparing relevant therapeutic options. ${ }^{41}{ }^{42}$ NMA does have limitations in the setting of systemic therapies for $\mathrm{AD}$, particularly differences in clinical trial design across included studies. Nevertheless, in the absence of head-to-head trial comparisons, the NMA approach provides comparative efficacy and safety data to aid decision making by clinicians and patients.

\section{Author affiliations}

${ }^{1}$ Division of Dermatology, Department of Medicine, University of Toronto, Toronto, Ontario, Canada

${ }^{2}$ Department of Medicine and Women's College Research Institute, Women's College Hospital, Toronto, Ontario, Canada

${ }^{3}$ School of Public Health, Brown University, Providence, Rhode Island, USA

${ }^{4}$ St John's Institute of Dermatology, Guy's and St Thomas' NHS Foundation Trust, London, UK

${ }^{5}$ Dermatology Centre, The University of Manchester, Salford Royal NHS Foundation Trust, Salford, UK

${ }^{6}$ Dutch Association for People with Atopic Dermatitis (VMCE), Nijkerk, The Netherlands

${ }^{7}$ Patient Representative (independent), Nottingham, UK

${ }^{8}$ Department of Dermatology, Academic Medical Centre, University of Amsterdam, Amsterdam, The Netherlands

${ }^{9}$ Center for Evidence-Based Healthcare, Technische Universitat Dresden

Medizinische Fakultat Carl Gustav Carus, Dresden, Germany

Contributors AMD contributed to study design and drafted the protocol manuscript. CF contributed to study design, drafted the PROSPERO protocol and provided critical revisions on the manuscript. AE, ZJ-L, ZZNY, BWMA, TB, PIS, DK and JS contributed to study design and provided critical revisions on the manuscript. PIS, DK, JS and CF are authors on a previous Cochrane systematic review on this topic. As this is a protocol paper, the research has not yet been conducted, and no data have been acquired or interpreted.

Funding The authors have not declared a specific grant for this research from any funding agency in the public, commercial or not-for-profit sectors.

Competing interests AMD: departmental research funding from Sanofi and Regeneron and consultancy for Sanofi, RTI Health Solutions and Eczema Society of Canada as well as Astellas Canada, Prime Inc, Spire Learning and the Eczema
Society of Canada. PIS: principal investigator (PI) Methotrexate versus Azathioprine for severe Atopic Dermatitis (MAcAD) trial, Pl of the Dutch national systemic therapy atopic eczema registry (TREAT NL) for adults and children. PS has served as a consultant to AbbVie, Anacor, Leo Pharma, Novartis and Sanofi, has received independent research grants ( $>4$ years ago) from Leo Pharma and Schering-Plough and has been involved in performing clinical trials with pharmaceutical industries that manufacture drugs used for the treatment of atopic dermatitis. JS: department research funding from Sanofi, Pfizer, ALK, Novartis and MSD. PI of the German national AE registry (TREAT Germany). CF: chief investigator (Cl) of the TREatment of severe Atopic eczema in children Trial (TREAT), a UK National Institute of Health Research-funded multicentre study comparing methotrexate and ciclosporin (www. treat-trial.org.uk). $\mathrm{Cl}$ of the UK national systemic therapy atopic eczema registry ( $\left.A^{\star} \mathrm{STAR}\right)$ for adults and children. Consultancy for Sanofi/Regeneron. All other team members have no conflict of interest to declare.

Patient consent Not required.

Provenance and peer review Not commissioned; externally peer reviewed.

Open access This is an open access article distributed in accordance with the Creative Commons Attribution Non Commercial (CC BY-NC 4.0) license, which permits others to distribute, remix, adapt, build upon this work non-commercially, and license their derivative works on different terms, provided the original work is properly cited, appropriate credit is given, any changes made indicated, and the use is non-commercial. See: http://creativecommons.org/licenses/by-nc/4.0/.

\section{REFERENCES}

1. Silverberg JI, Simpson EL. Associations of childhood eczema severity: a US population-based study. Dermatitis 2014;25:107-14.

2. Barbarot S, Auziere S, Gadkari A, et al. Epidemiology of atopic dermatitis in adults: Results from an international survey. Allergy 2018;73:1284-93.

3. Sidbury R, Davis DM, Cohen DE, et al. Guidelines of care for the management of atopic dermatitis: section 3. Management and treatment with phototherapy and systemic agents. J Am Acad Dermatol 2014;71:327-49.

4. Roekevisch E, Spuls PI, Kuester D, et al. Efficacy and safety of systemic treatments for moderate-to-severe atopic dermatitis: a systematic review. J Allergy Clin Immunol 2014:133:429-38.

5. Beck LA, Thaçi D, Hamilton JD, et al. Dupilumab treatment in adults with moderate-to-severe atopic dermatitis. $N$ Engl J Med 2014;371:130-9.

6. Paller AS, Kabashima K, Bieber T. Therapeutic pipeline for atopic dermatitis: End of the drought? J Allergy Clin Immunol 2017:140:633-43.

7. Blauvelt $A$, de Bruin-Weller M, Gooderham M, et al. Long-term management of moderate-to-severe atopic dermatitis with dupilumab and concomitant topical corticosteroids (LIBERTY AD CHRONOS): a 1-year, randomised, double-blinded, placebocontrolled, phase 3 trial. Lancet 2017;389:2287-303.

8. Simpson EL, Bieber T, Guttman-Yassky E, et al. Two phase 3 trials of dupilumab versus placebo in atopic dermatitis. $N$ Engl $J$ Med 2016;375:2335-48.

9. Thaçi D, Simpson EL, Beck LA, et al. Efficacy and safety of dupilumab in adults with moderate-to-severe atopic dermatitis inadequately controlled by topical treatments: a randomised, placebo-controlled, dose-ranging phase $2 \mathrm{~b}$ trial. Lancet 2016;387.

10. Goujon C, Viguier M, Staumont-Sallé $D$, et al. Methotrexate versus cyclosporine in adults with moderate-to-severe atopic dermatitis: a phase iii randomized noninferiority trial. J Allergy Clin Immunol Pract 2018;6.

11. Schram ME, Roekevisch E, Leeflang MM, et al. A randomized trial of methotrexate versus azathioprine for severe atopic eczema. J Allergy Clin Immunol 2011;128:353-9.

12. Guttman-Yassky E, Brunner PM, Neumann AU, et al. Efficacy and safety of fezakinumab (an IL-22 monoclonal antibody) in adults with moderate-to-severe atopic dermatitis inadequately controlled by conventional treatments: A randomized, double-blind, phase 2 a trial. J Am Acad Dermatol 2018;78:872-81.

13. Guttman-Yassky E, Silverberg JI, Nemoto O, et al. Baricitinib in adult patients with moderate-to-severe atopic dermatitis: a phase 2 parallel, double-blinded, randomized placebo-controlled multipledose study. J Am Acad Dermatol 2018.

14. Shamseer L, Moher D, Clarke M, et al. Preferred reporting items for systematic review and meta-analysis protocols (PRISMA-P) 2015: elaboration and explanation. BMJ 2015;349:g7647.

15. Chopra R, Vakharia PP, Sacotte R, et al. Severity strata for Eczema Area and Severity Index (EASI), modified EASI, Scoring Atopic 
Dermatitis (SCORAD), objective SCORAD, Atopic Dermatitis Severity Index and body surface area in adolescents and adults with atopic dermatitis. Br J Dermatol 2017;177:1316-21.

16. Leshem YA, Hajar T, Hanifin JM, et al. What the Eczema Area and Severity Index score tells us about the severity of atopic dermatitis: an interpretability study. Br J Dermatol 2015;172:1353-7.

17. Küster D, Spuls PI, Flohr C, et al. Effects of systemic immunosuppressive therapies for moderate-to-severe eczema in children and adults. Cochrane Database Syst Rev 2015;52.

18. Morrison A, Polisena J, Husereau D, et al. The effect of Englishlanguage restriction on systematic review-based meta-analyses: a systematic review of empirical studies. Int J Technol Assess Health Care 2012;28:138-44.

19. Rathbone J, Hoffmann T, Glasziou P. Faster title and abstract screening? Evaluating Abstrackr, a semi-automated online screening program for systematic reviewers. Syst Rev 2015;4:80.

20. Leahy J, O'Leary A, Afdhal N, et al. The Impact of Individual Patient Data in a Network Meta Analysis: An investigation into parameter estimation and model selection. Res Synth Methods 2018.

21. Schmitt J, Apfelbacher C, Spuls PI, et al. The Harmonizing Outcome Measures for Eczema (HOME) roadmap: a methodological framework to develop core sets of outcome measurements in dermatology. J Invest Dermatol 2015;135:24-30.

22. Schmitt J, Spuls PI, Thomas KS, et al. The Harmonising Outcome Measures for Eczema (HOME) statement to assess clinical signs of atopic eczema in trials. J Allergy Clin Immunol 2014;134:800-7.

23. Spuls PI, Gerbens LAA, Simpson E, et al. Patient-oriented eczema measure (poem), a core instrument to measure symptoms in clinical trials: a harmonising outcome measures for eczema (home) statement. Br J Dermatol 2017;176:979-84.

24. Rehal B, Armstrong AW, Armstrong A. Health outcome measures in atopic dermatitis: a systematic review of trends in disease severity and quality-of-life instruments 1985-2010. PLoS One 2011;6:e17520.

25. Schmitt J, Langan S, Deckert S, et al. Assessment of clinical signs of atopic dermatitis: a systematic review and recommendation. $J$ Allergy Clin Immunol 2013;132:1337-47.

26. Heinl D, Chalmers J, Nankervis $\mathrm{H}$, et al. Eczema trials: quality of life instruments used and their relation to patient-reported outcomes. a systematic review. Acta Derm Venereol 2016;96:596-601.

27. Furukawa TA, Barbui C, Cipriani A, et al. Imputing missing standard deviations in meta-analyses can provide accurate results. $J$ Clin Epidemiol 2006;59:7-10.
28. Caldwell DM, Ades AE, Higgins JP. Simultaneous comparison of multiple treatments: combining direct and indirect evidence. $B M J$ 2005;331:897-900.

29. Lu G, Ades AE. Combination of direct and indirect evidence in mixed treatment comparisons. Stat Med 2004;23:3105-24.

30. Salanti G. Indirect and mixed-treatment comparison, network, or multiple-treatments meta-analysis: many names, many benefits, many concerns for the next generation evidence synthesis tool. Res Synth Methods 2012;3:80-97.

31. Valkenhoef Gvan, Kuiper joel. gemtc: Network Meta-Analysis Using Bayesian MethodsVersion 0.8-2, 2016.

32. Dias S, Welton NJ, Sutton AJ, et al. A generalised linear modelling framework for pairwise and network meta-analysis of randomised controlled trials [internet]. 2014

33. Dias S, Welton NJ, Caldwell DM, et al. Checking consistency in mixed treatment comparison meta-analysis. Stat Med 2010;29(78):932-44.

34. Salanti G, Ades AE, loannidis JP. Graphical methods and numerical summaries for presenting results from multiple-treatment metaanalysis: an overview and tutorial. J Clin Epidemiol 2011;64:163-71.

35. Higgins JP, Altman DG, Gøtzsche PC, et al. The Cochrane Collaboration's tool for assessing risk of bias in randomised trials. BMJ 2011;343:d5928.

36. Higgins J, Green S. Cochrane handbook for systematic reviews of interventions version 5.1. 0. 2011: The Cochrane Collaboration, 2013.

37. Nikolakopoulou A, Mavridis D, Furukawa TA, et al. Living network meta-analysis compared with pairwise meta-analysis in comparative effectiveness research: empirical study. BMJ 2018;360:k585.

38. Elliott JH, Synnot A, Turner T, et al. Living systematic review: 1. Introduction - the why, what, when, and how. J Clin Epidemiol 2017; $91: 23-30$

39. Synnot A, Turner T, Elliott J. Cochrane Living Systematic Reviews Interim guidance for pilots, 2017.

40. Hutton B, Salanti G, Caldwell DM, et al. The PRISMA extension statement for reporting of systematic reviews incorporating network meta-analyses of health care interventions: checklist and explanations. Ann Intern Med 2015;162:777-84.

41. Sbidian E, Chaimani A, Garcia-Doval I, et al. Systemic pharmacological treatments for chronic plaque psoriasis: a network meta-analysis. Cochrane Database Syst Rev 2017;12:CD011535.

42. Jabbar-Lopez ZK, Yiu ZZN, Ward V, et al. Quantitative evaluation of biologic therapy options for psoriasis: a systematic review and network meta-analysis. J Invest Dermatol 2017;137:1646-54. 